\title{
The Double-Time Protein Kinase Regulates the Subcellular Localization of the Drosophila Clock Protein Period
}

\author{
Shawn A. Cyran, ${ }^{1}$ Georgia Yiannoulos, ${ }^{1}$ Anna M. Buchsbaum, ${ }^{1}$ Lino Saez, ${ }^{2}$ Michael W. Young, ${ }^{2}$ and Justin Blau ${ }^{1}$ \\ ${ }^{1}$ Department of Biology, New York University, New York, New York 10003, and ${ }^{2}$ The Rockefeller University, New York, New York 10021
}

The Period (PER), Timeless (TIM), and Double-Time (DBT) proteins are essential components of one feedback loop in the Drosophila circadian molecular clock. PER and TIM physically interact. Coexpression of PER and TIM promotes their nuclear accumulation and influences the activity of DBT: although DBT phosphorylates and destabilizes PER, this is suppressed by TIM. Experiments using Drosophila cells in culture have indicated that PER can translocate to the nucleus without TIM and will repress transcription in a DBT-potentiated manner. In this study, we examined the control of PER subcellular localization in Drosophila clock cells in vivo. We found that PER can translocate to the nucleus in tim $^{01}$ null mutants but only if DBT kinase activity is inhibited. We also found that nuclear PER is a potent transcriptional repressor in $d b t$ mutants in vivo without TIM. Thus, in vivo, DBT regulates PER subcellular localization, in addition to its previously documented role as a mediator of PER stability. However, DBT does not seem essential for transcriptional repression by PER. It was reported previously that overexpression of a second kinase, Shaggy (SGG)/Glycogen Synthase Kinase 3, accelerates PER nuclear accumulation. Here, we show that these effects of SGG on PER nuclear accumulation require TIM. We propose a revised clock model that incorporates this tight kinase regulation of PER and TIM nuclear entry.

Key words: circadian; Period; Double-Time; Timeless; nuclear entry; Drosophila

\section{Introduction}

Rhythms of gene expression and protein levels lie at the core of the molecular clocks that regulate circadian $(\sim 24 \mathrm{~h})$ rhythms of behavior and physiology. The best-studied circadian feedback loop involves the Drosophila clock genes period (per) and timeless ( $\mathrm{tim}$ ). Many components of this negative feedback loop play similar roles in the mammalian clock (Panda et al., 2002; Stanewsky, 2003).

In Drosophila, per and tim transcription is activated by Clock (CLK)/Cycle (CYC) heterodimers binding to E-boxes in the per and tim promoters. PER and TIM proteins heterodimerize in the cytoplasm, and, until recently (see below), it was believed that they enter the nucleus together. PER is more stable than TIM in the nucleus and represses additional per and tim transcription by directly inhibiting CLK/CYC.

The PER/TIM loop is characterized by delays that separate transcription from repression, important for rhythmic, as op-

\footnotetext{
Received Jan. 19, 2005; revised April 25, 2005; accepted April 28, 2005.

Most of this investigation was conducted in a facility constructed with support from Research Facilities Improvement Grant C06 RR-15518-01 from the National Center for Research Resources-National Institutes of Health (NIH). Work in the authors' laboratories was supported by NIH Grants GM54339 (M.W.Y.) and GM063911 (J.B.). We are very grateful to Jae Park, Paul Taghert, and the Bloomington Stock Center for providing antibodies and flies. Antibodies to ELAV, developed by Gerry Rubin, were obtained from the Developmental Studies Hybridoma Bank under the auspices of the National Institute of Child Health and Human Development and maintained by the Department of Biological Sciences, University of lowa (lowa City, IA). We also thank Sue Chong and Meg Younger for excellent technical assistance and Ben Collins, Mark Drapeau, and Claude Desplan for invaluable comments on this manuscript.

Correspondence should be addressed to Justin Blau, Department of Biology, New York University, 100 Washington Square East, New York, NY 10003. E-mail: justin.blau@nyu.edu.

G. Yiannoulos' present address: Veterans Affairs Medical Center, 130 West Kingsbridge Road, Bronx, NY 10468. DOI:10.1523/JNEUROSCI.0263-05.2005

Copyright $\odot 2005$ Society for Neuroscience $\quad$ 0270-6474/05/255430-08\$15.00/0
}

posed to continuous, negative feedback. Peak per RNA and protein levels are separated by $\sim 6 \mathrm{~h}$ at least partly by Double-Time $(\mathrm{DBT}) /$ Casein Kinase $1 \epsilon(\mathrm{CK} 1 \epsilon)$, a protein kinase that destabilizes cytoplasmic PER while TIM levels are low (Price et al., 1998; Suri et al., 2000). Thus, PER levels are constitutively low in tim $^{01}$ mutants (Price et al., 1995). DBT stably associates with PER (Kloss et al., 2001) and phosphorylates PER (Price et al., 1998; Ko et al., 2002) (S. Kivimae and M. W. Young, unpublished data), which leads to PER ubiquitination and degradation (Grima et al., 2002; Ko et al., 2002). These in vivo findings led to the model that rising TIM levels allow the formation of a DBT-resistant PER/ TIM complex (Kloss et al., 1998, 2001; Price et al., 1998). DBT also regulates the stability of nuclear PER, consistent with the identification of stable PER/DBT complexes after TIM degradation in the early morning (Kloss et al., 2001) and by altered nuclear PER stability in $d b t$ mutants (Price et al., 1998).

Stabilized PER fusion proteins are cytoplasmic in $\operatorname{tim}^{01} \mathrm{mu}-$ tants (Vosshall et al., 1994), and, reciprocally, TIM accumulates in the cytoplasm of per $^{\circ}$ mutants (Hunter-Ensor et al., 1996; Myers et al., 1996), leading to the model that PER and TIM enter the nucleus together. The timing of PER and TIM nuclear entry is tightly regulated, at least in the pacemaker lateral neurons (LNs), and is promoted by the Shaggy (SGG) and CK2 kinases (Martinek et al., 2001; Lin et al., 2002; Akten et al., 2003).

However, certain features of this Drosophila clock model have been questioned. Shafer et al. $(2002,2004)$ found PER in the nuclei of LNs before TIM, and, in some Drosophila S2 cell studies, transfected PER repressed CLK/CYC activity without cotransfected TIM (Chang and Reppert, 2003; Weber and Kay, 2003; Nawathean and Rosbash, 2004). Therefore, PER nuclear accumulation may not simply depend on heterodimerization with TIM. 
We therefore initiated an additional study of PER localization in clock cells in vivo. Here, we show that DBT kinase activity normally inhibits nuclear accumulation of PER in pacemaker neurons and adult photoreceptor cells: in the absence of both DBT kinase activity and TIM, PER enters the nucleus and represses transcription. We propose a revised Drosophila clock model based on properties of clock proteins established in vivo.

\section{Materials and Methods}

Fly strains and immunocytochemistry. Larvae and flies were entrained in standard light/dark cycles or were mainly taken from unentrained conditions if in an arrhythmic tim ${ }^{01}$ background. yellow white $(y w)$ larvae and flies were used as wild-type controls. Upstream activation sequence (uas) - sggB flies were described by Bourouis (2002). SG3 flies were originally described by Vosshall et al. (1994). The SG3 transgene uses the per promoter to drive the expression of a fusion protein of the first 636 aa of PER fused to LacZ. Staining of whole brains and of adult head sections was as described previously (Myers et al., 1996; Price et al., 1998; Kloss et al., 2001). Antibodies to PER were provided by Ralf Stanewsky and Jeff Hall. Rat and guinea pig antibodies to Pigment-Dispersing Factor (PDF) were provided by Jae Park and Paul Taghert, respectively. Mouse antiPDF antibodies were generated against amidated PDF peptide (NSELINSLLSLPKNMNDA-NH ${ }_{2}$ ) by PickCell Laboratories (Amsterdam, The Netherlands), and antibodies to LacZ were from Promega (Madison, WI). For adult head sections, PER antibody was first preabsorbed against $y w$ embryos and then against acetone powder made from per ${ }^{0}$ flies.

Western blots, immunoprecipitation, and RNA analysis. Western blots were as described previously (Cyran et al., 2003), except that only $2 \mu \mathrm{g}$ of protein extracts were used to test SGG levels. Affinity-purified DBT antibodies were described by Kloss et al. (2001), and antibodies to SGG/ Glycogen Synthase Kinase 3 (GSK3) were purchased from Upstate Biotechnology (Lake Placid, NY). Immunoprecipitations with anti-DBT were performed as described previously (Kloss et al., 2001).

RNA levels were analyzed by quantitative real-time PCR as in the study by Cyran et al. (2003), except that $t i m^{01}$ and $t i m^{01}$; $d b t^{a r}$ flies were maintained in constant light. Primer combinations for vrille (vri) and Par domain protein $1 \epsilon(P d p 1 \epsilon)$ were described previously by Cyran et al. (2003). Primers (TIB Molbiol, Adelphia, NJ) used were as follows: tim forward, ATGGTGGCTCTGATGAG; tim reverse, CCAAAGAGACATTGTCGC; tim Hybridization Probe 1 (HP1), AGTCCTCGTTCGAGCGG-fluorescein; tim HP2, Red640-GGAGGTAAACGGATCGCACT; $c y c$ forward, TTCGCAACTCCACAGTAC; $c y c$ reverse, AGGGATTCTTGAAGGCC; $c y c$ HP1, GGGCAAGTTCCTGTTCATAGACC-fluorescein; cyc HP2, Red640-CGTGCCACCCTCGTGA.

\section{Results}

\section{PER enters the nucleus without TIM in $\boldsymbol{d b t}^{P}$ mutants}

PER is constitutively located in the nucleus of the pacemaker LNs in $d b t^{P}$ single mutants, which produce very low levels of $d b t$ RNA (Kloss et al., 1998; Price et al., 1998). Because DBT was proposed to destabilize cytoplasmic PER (Price et al., 1998), one prediction was that PER should accumulate to high levels in the cytoplasm of clock cells in the absence of both TIM and DBT. Because $d b t^{P}$ mutants die during pupation (Price et al., 1998), PER localization was tested in the pacemaker LNs of third instar larvae doubly mutant for $t^{i m}{ }^{01}$ and $d b t^{P}$. These cells are the precursors to the adult small- $\mathrm{LN}_{\mathrm{v}} \mathrm{s}\left(\mathrm{s}-\mathrm{LN}_{\mathrm{v}} \mathrm{s}\right)$, which are essential for adult circadian behavior. The LNs were identified with an antibody to the PDF neuropeptide. PER was located in the nucleus of the LNs in $d b t^{P}$ single mutants, as observed previously (Fig. 1) (Price et al., 1998). Surprisingly, we also observed that PER was located in the nucleus of the LNs in $\operatorname{tim}^{01} ; d b t^{P}$ double mutants (Fig. 1). Therefore, PER can enter and accumulate in the nucleus in the absence of TIM when DBT protein levels are significantly lower than in wild type. These in vivo results are in contrast to the in vitro results of

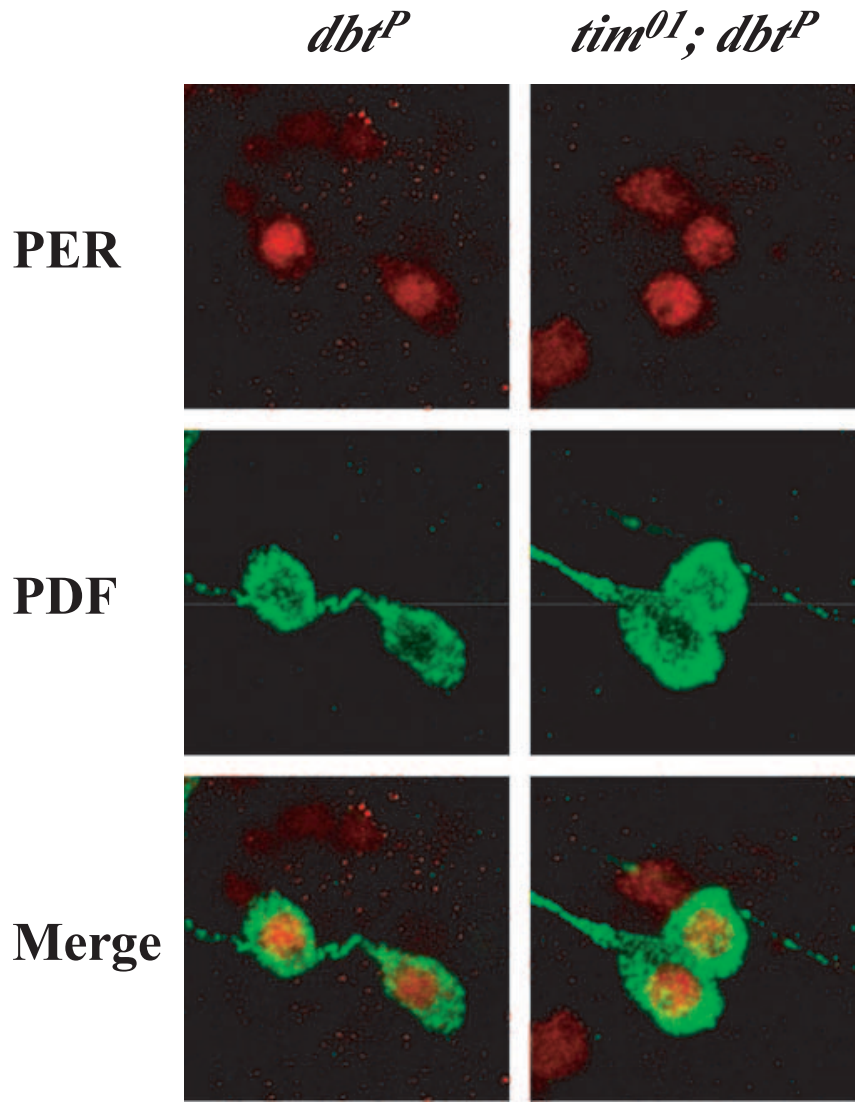

Figure 1. PER enters the nucleus independently of TIM in a $d b t^{P}$ background. Third instar larval brains were stained with antibodies to PER (red; top panels) and PDF (green; middle panels). Red and green images are shown merged in the bottom panels. PDF marks the cell body and axons of pacemaker neurons, the LNs. PER was detected in the nuclei of larval LNs in $d b t^{P}$ single mutants (left) and $t^{2}{ }^{01} ; d b t^{p}$ double mutants (right). Essentially identical results were seen in at least 50 brain hemispheres for each genotype.

Nawathean and Rosbash (2004), in which inactivation of $d b t$ by RNA interference (RNAi) in Drosophila cultured cells did not increase nuclear accumulation of PER.

\section{DBT kinase activity regulates nuclear entry of PER}

DBT has two previously established activities toward PER: it forms a stable complex with PER in vivo (Kloss et al., 2001), and it promotes PER phosphorylation in both the cytoplasm and the nucleus (Price et al., 1998; Ko et al., 2002). These two functions are effectively removed by the hypomorphic $d b t^{P}$ mutation. To test whether physical association between DBT and PER, or DBT kinase activity for PER, normally prevents PER from accumulating in the nucleus, we used a point mutation in $d b t\left(d b t^{a r}\right)$, originally identified and characterized by Rothenfluh et al. (2000c).

$\mathrm{DBT}^{\mathrm{AR}}$ protein was detected at levels indistinguishable from wild-type DBT (Fig. 2A) (Rothenfluh et al., 2000c), and affinitypurified antibodies to DBT coprecipitated similar amounts of PER in extracts isolated from the heads of either adult wild-type flies or $t^{01}{ }^{01}$; $d b t^{a r}$ double-mutant flies (Fig. $2 B$ ). These results indicate that, like $\mathrm{DBT}^{+}$(Kloss et al., 2001), $\mathrm{DBT}^{\mathrm{AR}}$ can stably associate with PER.

The $d b t^{a r}$ mutation causes a histidine-to-tyrosine change in the highly conserved catalytic domain of the enzyme (Rothenfluh et al., 2000c), and this renders DBT ${ }^{A R}$ a very poor kinase for PER in vitro (Ko et al., 2002) (Kivimae and Young, unpublished data). Consistent with this, most of the PER in extracts isolated from 


\section{A Western}

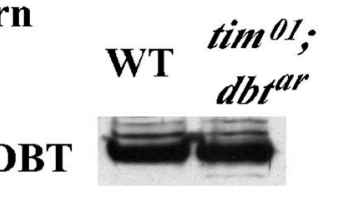

B IP $\alpha$ DBT

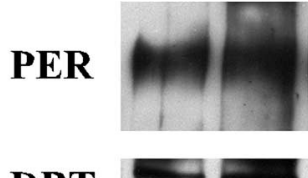

DBT

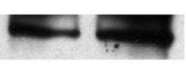

C Western

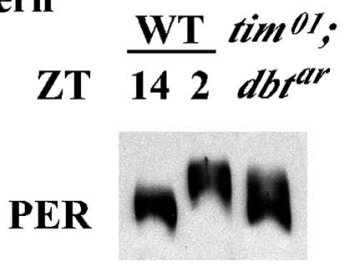

Figure 2. PER stably associates with $D B T^{A R}$ but is mostly hypophosphorylated. $A$, Western blots of DBT protein from adult head extracts of control wild-type flies (WT;ZT23; left) and tim ${ }^{01}$; $d b t^{a r}$ double-mutant flies (right). DBT ${ }^{+}$and DBT ${ }^{\mathrm{AR}}$ proteins accumulated to similar levels. The result shown here is representative of three experiments and is consistent with the results of Rothenfluh et al. (2000c). B, An affinity-purified anti-DBT antibody was used to immunoprecipitate (IP) DBT from head extracts of wild-type flies (ZT23; left lane) and $\mathrm{tim}^{01} ; \mathrm{dbt}^{\mathrm{ar}}$ double-mutant flies (right lane). Immunoprecipitates were then subjected to Western blotting with anti-PER (top) and anti-DBT (bottom) antibodies. PER was immunoprecipitated by anti-DBT antibodies in both wild-type and $\mathrm{tim}^{01}$; $\mathrm{db}^{\mathrm{ar}}$ double-mutant flies. The results shown are representative of three experiments. C, Head extracts of wild-type flies from ZT14 (left lane) and ZT2 (middle lane) were run alongside extracts from $\mathrm{tim}^{01}$; $\mathrm{dbt}^{a r}$ double-mutant flies (right lane) and blotted for PER protein. Because $\operatorname{tim}^{01}$; $d b^{a r}$ double-mutant flies are arrhythmic, they were not entrained. PER shows a characteristic difference in mobility in wildtype flies between ZT14 and ZT2, with the decreased mobility at ZT2 attributable to increased PER phosphorylation (Edery etal., 1994). Neither of these times correspond to peak PER levels in wild-type flies but were chosen as extremes in PER phosphorylation status. PER protein extracted from $\operatorname{tim}^{01}$; $d b t^{a r}$ double-mutant flies was mostly hypophosphorylated, although some slower mobility PER was detected. The results shown are representative of three experiments.

$t^{i m^{01}}$; $d b t^{a r}$ double-mutant flies had an electrophoretic mobility similar to the hypophosphorylated form of PER typically seen when PER first begins to accumulate in wild-type flies at Zeitgeber time (ZT) 14 (Zeitgeber time is the time in a $12 \mathrm{~h}$ light/dark cycle) (Fig. 2C), which is in contrast to the highly phosphorylated forms of PER seen in the early morning (ZT2). In conclusion, $\mathrm{DBT}^{\mathrm{AR}}$ apparently binds PER normally but is mostly defective in phosphorylating PER, in agreement with the previous genetic interactions of $d b t^{a r}$ with some per alleles (Rothenfluh et al., 2000c).

Subsequently, we used the $d b t^{a r}$ mutation to test whether DBT binding to PER or DBT phosphorylation of PER regulates PER subcellular localization. Because $\sim 5 \%$ of $d b t^{a r}$ homozygotes survive to adulthood (Rothenfluh et al., 2000a), PER localization was tested in the adult s-LN $\mathrm{LN}_{\mathrm{v}}$ s. Wild-type (ZT23), tim ${ }^{01}$; $d b t^{a r}$ doublemutant, and tim $^{01}$ single-mutant brains were stained with antibodies to PER and PDF. The results in Figure 3 revealed that PER was detected in the nucleus of the s- $\mathrm{LN}_{\mathrm{v}} \mathrm{s}$ of wild-type and $\mathrm{tim}^{01}$; $d b^{a r}$ double mutants but was undetectable in $\mathrm{tim}^{01}$ single mutants.

We also tested PER localization in peripheral clock cells of $t{ }^{01}{ }^{01} ; d b t^{a r}$ double mutants. We used photoreceptor cells, be-

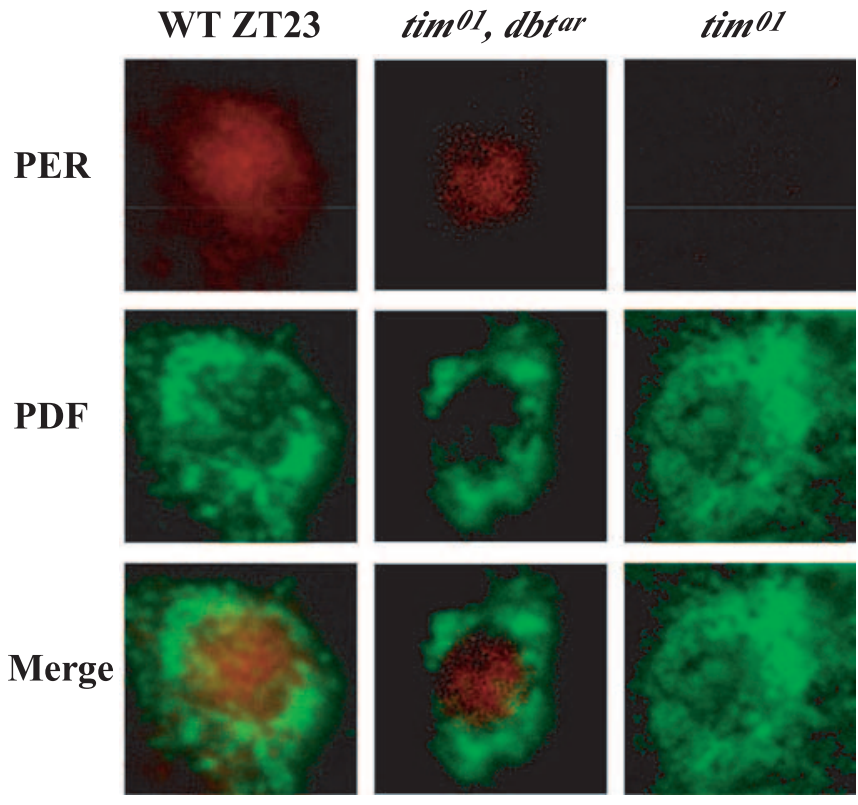

Figure 3. PER enters the nucleus of adult pacemaker neurons independently of TIM in a dbt ${ }^{a r}$ background. Brains of adult wild-type flies (WT; ZT23; left panels), tim ${ }^{01}$; $\mathrm{db}^{\mathrm{ar}}$ double-mutant flies (middle panels), and tim ${ }^{01}$ single-mutant flies (right panels) were stained as in Figure 1 with antibodies to PER (red) and PDF (green). PER accumulated in nuclei of wild-type and tim ${ }^{01}$; $d b t^{a r}$ double-mutant $s-\mathrm{LN}_{\mathrm{v}}$ s but was undetectable in a $\operatorname{tim}^{01}$ single-mutant background. Essentially identical results were seen in at least 20 brain hemispheres from each genotype.

cause they contain functional molecular clocks. The cell nuclei of all neurons (including photoreceptors) were marked with an antibody that recognizes the Embryonic Lethal Abnormal Vision protein (ELAV) (Robinow and White, 1991). The results in Figure 4 show that PER was localized to the nuclei of photoreceptor cells in wild-type (ZT23) and in $t i^{01}$; $d b t^{a r}$ double mutants but was undetectable in $\operatorname{tim}^{01}$ or $\mathrm{per}^{0}$ single mutants (Fig. 4). Our results are consistent with previous data showing that PER is constitutively nuclear in the photoreceptor cells of $d b t^{a r}$ single mutants (Rothenfluh et al., 2000c), and our findings here reveal that nuclear PER accumulation in $d b t^{a r}$ mutants is independent of TIM. The presence of PER in the nuclei of central and peripheral clock cells that synthesize wild-type levels of a kinasedefective DBT protein indicates that DBT kinase activity, rather than a simple DBT-PER protein interaction, normally prevents PER from accumulating in the nucleus in the absence of TIM. Thus, PER, if hypophosphorylated and not degraded, translocates to the nucleus, indicative of its intrinsic properties.

\section{A stable PER fusion protein associates with nuclei in the} absence of tim and wild-type $d b t$

Nuclear PER in $t \mathrm{tim}^{01} ; d b t^{P}$ double mutants could simply reflect that high levels of PER in pacemaker neurons are sufficient to override the normal block to PER nuclear entry in the absence of TIM. However, the levels of PER detected in $\operatorname{tim}^{01}$; $d b t^{a r}$ doublemutant pacemaker cells are not as high as those in wild type, and yet, PER is nuclear (Figs. 3, 4). This observation suggests that nuclear PER can repress the transcription of clock genes in the absence of TIM (directly examined below). Furthermore, levels of PER in the Western blots of $t t^{01}$; $d b t^{a r}$ head extracts are within the normal physiological range of PER from wild-type flies (Fig. 2C). Thus, it is unlikely that nuclear PER is simply a consequence of increased PER stability. Instead, PER nuclear accumulation in 
A

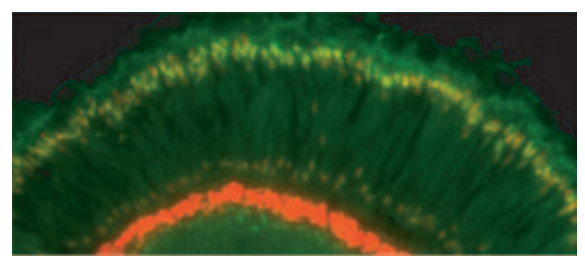

$\operatorname{tim} 01$

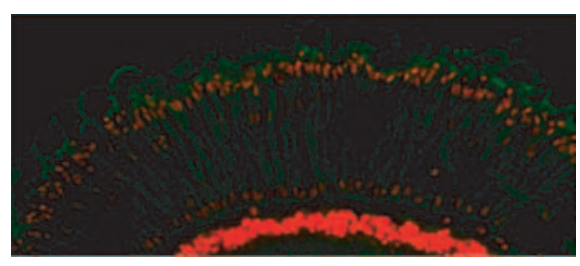

B

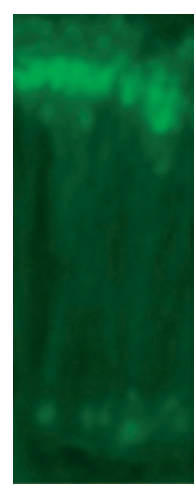

WT
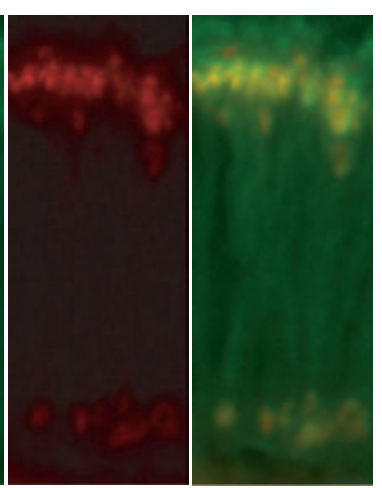

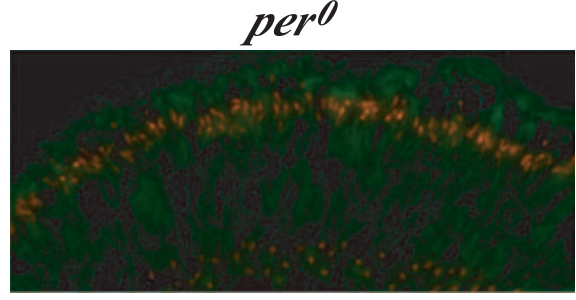

tim01; dbtar

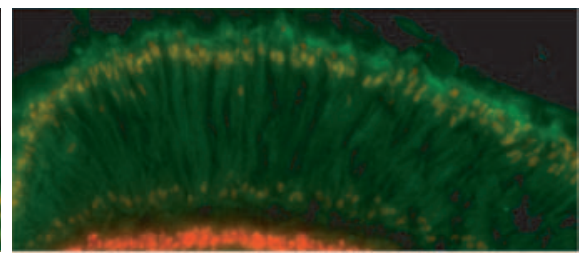

perl

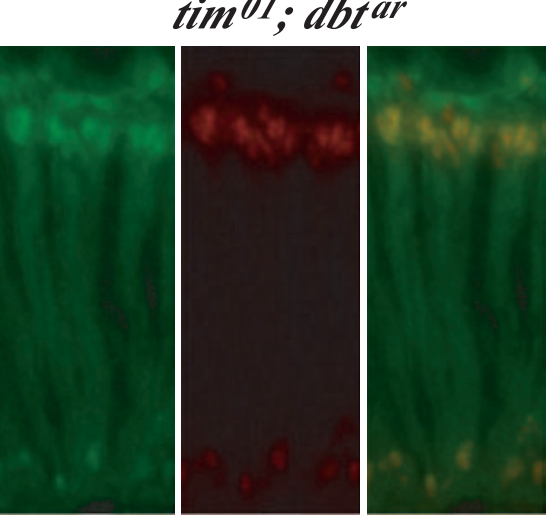

Figure 4. PER enters photoreceptor cell nuclei independently of TIM in a $d b t^{a r}$ mutant background. $A$, Sections through adult heads from wild type (WT; ZT23; top left), $\operatorname{tim}^{01}$; $\mathrm{db}^{a r}$ double mutants (top right), tim ${ }^{01}$ single mutants (bottom left), and per ${ }^{0}$ single mutants (bottom right) stained with antibodies to PER (green) and ELAV (red). PER localized to the nucleus in wild-type and $\operatorname{tim}^{01} ; \mathrm{db}^{a r}$ double-mutant flies (seen more clearly at higher magnification in $\boldsymbol{B}$ ) but was undetectable in $\operatorname{tim}^{01}$ and $\mathrm{per}^{0}$ single mutants. Essentially identical results were seen in sections from at least 25 different adult heads from each genotype.

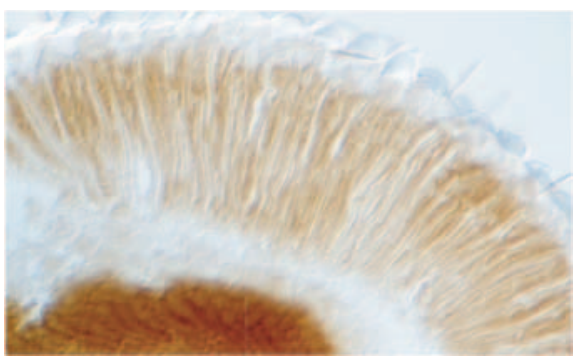

$\operatorname{tim}^{01}, S G 3 ; d b t^{a r} /+$

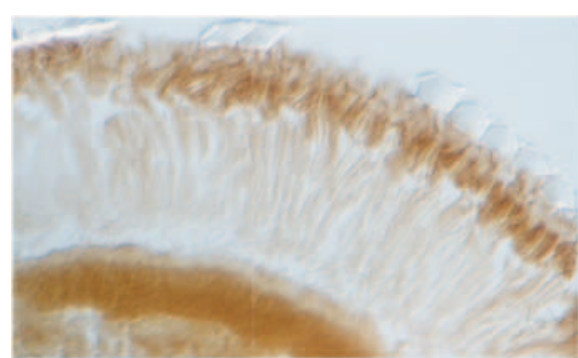

$\operatorname{tim}^{01}, S G 3 ; d b t^{a r}$

Figure 5. The subcellular distribution of a stable PER fusion protein, $\mathrm{SG} 3$, is also controlled by $\mathrm{dbt}$. Sections through adult heads of $t \mathrm{tm}^{01}, \mathrm{SG3} ; \mathrm{dbt}^{a r} /+$ (left) and $t \mathrm{tm}^{01}, \mathrm{SG3}$; $d b t^{a r}$ (right) flies stained with antibodies to LacZ and visualized via a DAB color reaction. SG3 was detected throughout the long cell bodies of the photoreceptors in $t_{i m}{ }^{01}$ flies heterozygous for $\mathrm{dbt}^{\mathrm{ar}}$, as originally seen in tim ${ }^{01}$ mutants (Vosshall et al., 1994). However, SG3 localizes to the periphery of the photoreceptor cells (where the nuclei are located) in $t^{01}{ }^{01} ; d b t^{a r}$ double mutants. Essentially identical results were seen in three independent experiments.

$\operatorname{tim}^{01} ; d b t^{P}$ and $\operatorname{tim}^{01} ; d b t^{a r}$ double-mutant clock cells presumably reflects the default state of PER in the absence of its regulator, DBT.

To test these ideas more extensively, we used the SG3 transgene in which the per promoter drives the expression of a cDNA encoding the first half of PER protein (amino acids 1-636) fused to LacZ (Vosshall et al., 1994). The SG3 protein is stable in a tim $^{01}$ background, unlike full-length PER, and was used previously to demonstrate that PER nuclear accumulation depends on TIM, because SG3 was nuclear in an otherwise wild-type background but cytoplasmic in a tim $^{01}$ background (Vosshall et al., 1994). SG3 contains a sufficient amount of PER to interact stably with DBT, and this interaction is independent of TIM (Kloss et al., 2001).

We used an antibody to LacZ to test the subcellular localization of SG3 in $\mathrm{tim}^{01}$; $d \mathrm{dt}^{\mathrm{ar}}$ double-mutant photoreceptor cells. The results in Figure 5 show a dramatic redistribution of SG3 between tim $^{01}$ single mutants and $t \mathrm{~m}^{01} ; d b t^{a r}$ double mutants. SG3 was widely distributed throughout the cytoplasm of the photoreceptor cells in tim $^{01}$ single mutants as originally observed (Vosshall et al., 1994). In contrast, SG3 was located at the periphery of the photoreceptor cells (where the nuclei are localized) in tim $^{01}$; $d b t^{a r}$ double mutants. Thus, the subcellular localization of this stabilized PER fusion protein is controlled by DBT kinase activity, and this suggests that DBT kinase activity regulates wild-type PER subcellular localization, in addition to its effects on PER stability. We infer that the detection of PER in the nucleus of $t \mathrm{tm}^{O 1}$; $d b t^{P}$ and $t{ }^{01}$; $d b t^{a r}$ clock cells is not a simple consequence of increased PER stability but instead reflects that DBT kinase activity opposes the tendency of PER to enter the nucleus and normally prevents premature PER nuclear localization.

\section{Nuclear PER represses CLK/CYC activity without TIM}

Previous studies have suggested that PER rather than PER/TIM inhibits CLK/CYCactivated transcription (Rothenfluh et al., 2000a; Chang and Reppert, 2003; Weber and Kay, 2003; Nawathean and Rosbash, 2004). Constitutive nuclear PER in $\mathrm{tim}^{\mathrm{O}}$; $d b t^{a r}$ mutant flies allowed CLK/CYC activity to be quantitatively measured in vivo in the complete absence of TIM. We assayed the levels of three genes directly activated by CLK/CYC: tim, vri, and Pdpl€ (Allada et al., 1998; Darlington et al., 1998; Rutila et al., 1998a; Blau and Young, 1999; McDonald and Rosbash, 2001; Cyran et al., 2003). tim, vri, and Pdpl $\epsilon$ levels were compared in RNA isolated from tim $^{01}$ single mutants and $\mathrm{tim}^{\mathrm{OI}}$; $d b t^{\mathrm{ar}}$ double-mutant adult fly heads. The results in Figure 6 show that steady-state RNA levels of all three genes were approximately fivefold lower in $\mathrm{tim}^{01} ; d b t^{a r}$ than in $\mathrm{tim}^{01}$ mutants.

These effects on PER target gene transcription probably indicate direct repression of CLK/CYC activity rather than an indirect effect of nuclear PER on CLK protein levels, because Clk RNA levels are approximately threefold higher in $t \mathrm{tm}^{01}$; $d b t^{a r}$ than in 


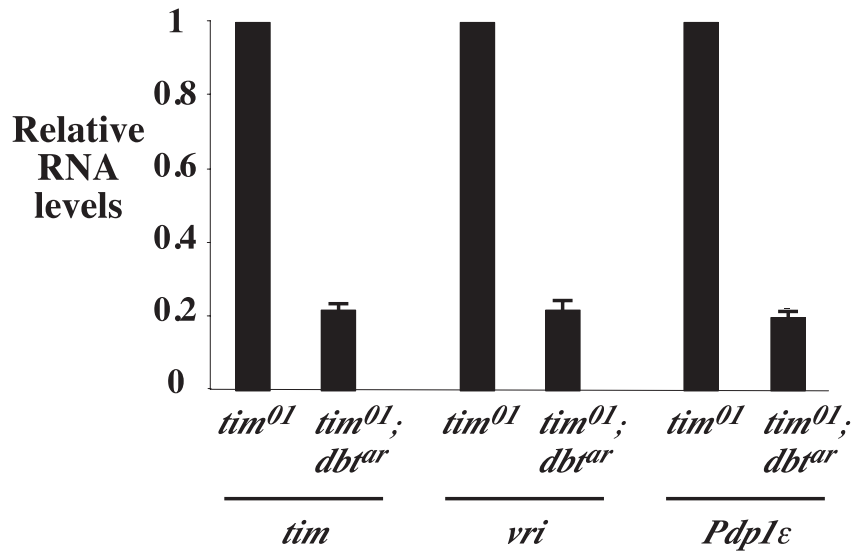

Figure 6. Nuclear PER represses CLK/CYC-activated transcription without TIM. RNA was extracted from $t^{01}{ }^{01}$ and $t i^{01}$; $d b t^{a r}$ adult fly heads, and levels of $t i m, v r i$, and Pdp $1 \epsilon$ were compared with cyc RNA levels by quantitative PCR, as described previously (Cyran et al., 2003). RNA levels in tim ${ }^{01}$ fly heads were set to 1 in each of two independent experiments and compared with RNA levels in $\operatorname{tim}^{01}$; $d b t^{a r}$ flies. The data are shown as the average, with error bars indicating the SD. Levels of tim, vri, and Pdp $1 \in$ RNA were approximately fivefold lower in tim $^{01}$; $d b t^{a r}$ than in $t^{2 m^{01}}$ fly heads. Similar results were also seen in one additional experiment analyzed by ribonuclease protection.

tim $^{01}$ mutants (J. Blau, unpublished data). Indeed, tim and $P d p 1 \epsilon$ RNA levels were essentially identical to their levels in wild-type flies at ZT2, the trough of their normal RNA cycle, indicating that their transcription is maximally repressed (Blau, unpublished data). Thus, nuclear PER represses CLK/CYC-activated transcription in the absence of TIM.

These effects of PER on CLK/CYC activity, independently of TIM, are consistent with repression of tim promoter activity in $t_{i m}{ }^{01}$; $d b t^{a r}$ double-mutant flies (Weber and Kay, 2003). Our results, and those of Weber and Kay (2003), also show that wildtype DBT activity is not required for transcriptional repression by PER. This conclusion is consistent with the constitutive repression of CLK/CYC activity (measured via tim RNA levels) in constant darkness in vivo in $d b t^{P}$ larval LNs (Price et al., 1998). However, these in vivo findings differ from those of Nawathean and Rosbash (2004), whose studies of Drosophila S2 cells in vitro (in which RNAi was used to remove DBT) indicated that DBT is required for repression by ectopically expressed PER. Evidently, S2 cells, which do not possess endogenous circadian clocks, do not provide an accurate model for certain steps of the in vivo mechanism, even when programmed to express clock genes and proteins.

\section{Overexpressing SGG has no effect on PER nuclear localization or stability without TIM}

Our results indicate that DBT kinase activity helps separate the phases of transcription and repression of CLK/CYC-activated genes by preventing premature PER accumulation in the nucleus. However, PER does enter the nucleus in wild-type clock cells around the middle of the night. This is generally considered to require TIM (Vosshall et al., 1994), and the precise timing of PER and TIM nuclear localization is affected by mutations in per, tim, $d b t, C K 2 \alpha, C K 2 \beta$, and $s g g$ (Curtin et al., 1995; Rothenfluh et al., 2000b; Bao et al., 2001; Martinek et al., 2001; Lin et al., 2002; Akten et al., 2003). Overexpression of $s g g$ in clock cells shortens the behavioral period and advances the timing of PER and TIM nuclear localization, presumably opposing the effect of DBTmediated phosphorylation. The period-altering effects of overexpressing $s g g$ were correlated with increased TIM phosphorylation in vivo (Martinek et al., 2001). However, because SGG/GSK3 and members of the Casein Kinase I family (including CKI $\epsilon$, mammalian DBT) have been shown to often phosphorylate the same substrate (Jia et al., 2002; Price and Kalderon, 2002; Okamura et al., 2004), we tested whether SGG could alter PER subcellular localization and/or stability independently of TIM.

sgg was overexpressed specifically in clock cells using the tim(UAS)-gal4 driver (Blau and Young, 1999) in a tim $^{01}$ background. We chose a uas-sggB transgene on the third chromosome (Bourouis, 2002), because it had the strongest effect on the period of adult locomotor rhythms of the sgg transgenes that we tested in a tim $^{+}$background, shortening the period to $18.5 \mathrm{~h}$ (A. M. Buchsbaum and Blau, unpublished data). The results in Figure $7 A$ show that PER was undetectable in the LNs of third instar larvae that overexpress $\mathrm{sgg}$ in a $\mathrm{tim}^{01}$ background just as in tim $^{01}$ mutants without increased sgg expression (Fig. 7A). Western blots from fly head extracts isolated from $\operatorname{tim}^{01}$ mutant flies overexpressing sgg also did not show altered PER levels or mobility compared with tim $^{01}$ mutants, indicating no change in PER stability or phosphorylation (Fig. $7 B$ ). In contrast, previous work has shown that TIM is hyperphosphorylated in response to overexpression of $s g g$ in a per $^{\circ}$ background (Martinek et al., 2001). Therefore, we conclude that the effects of SGG on the Drosophila circadian clock require tim. Our results provide additional evidence for a functional interaction of SGG and TIM and are consistent with the finding that TIM can be directly phosphorylated by mammalian SGG (GSK3) in vitro (Martinek et al., 2001).

\section{Discussion}

The results presented in this study reveal a novel function for DBT in the Drosophila circadian clock: DBT kinase activity controls the subcellular localization of PER. Phosphorylated PER is retained in the cytoplasm, whereas hypophosphorylated PER can accumulate in nuclei. Hypophosphorylated PER does not require TIM for access to the nucleus; however, as discussed below, TIM plays an important role in regulating DBT-dependent phosphorylation of PER. A model depicting our current view of the Drosophila clockworks is depicted in Figure 8.

Our results may provide a mechanistic explanation for the findings of Bao et al. (2001), who showed that the mutant DBT $^{S}$ protein delayed nuclear accumulation of PER in photoreceptor cells. Delayed PER nuclear entry would be expected if DBT ${ }^{\mathrm{S}}$ elevates PER phosphorylation, and in vitro studies by Ko et al. (2002) found that $\mathrm{DBT}^{\mathrm{S}}$ is a more active PER kinase than wildtype DBT. However, there may be a more important qualitative shift in kinase activity in $\mathrm{DBT}^{\mathrm{S}}$ that produces this PER response. An independent study (Preuss et al., 2004) found that engineering the $\mathrm{DBT}^{\mathrm{S}}$ mutation into Xenopus $\mathrm{CK} 1 \delta$ reduced phosphorylation of another substrate, casein, and Bao et al. (2001) found that $\mathrm{DBT}^{\mathrm{S}}$ delayed nuclear accumulation of PER without affecting its stability; wild-type or higher levels of PER protein accumulation were observed in $d b t^{S}$ mutants. Thus, as originally suggested by Bao et al. (2001), $\mathrm{DBT}^{\mathrm{S}}$ might produce a specific change in the pattern of PER phosphorylation that promotes cytoplasmic retention of PER without affecting stability.

$\mathrm{CK} 1 \epsilon$, the vertebrate DBT ortholog, has been shown to phosphorylate mammalian PER (Lowrey et al., 2000; Toh et al., 2001). CK1 $\epsilon$ also affects the subcellular localization of mammalian PER proteins. However, independent studies differ, finding that CK1 $\epsilon$ alternatively promotes or retards PER nuclear accumulation, depending on the cell line used (Vielhaber et al., 2000; Akashi et al., 2002; Miyazaki et al., 2004; Takano et al., 2004). These studies all monitored PER transfected into mammalian cell lines, and the 
A

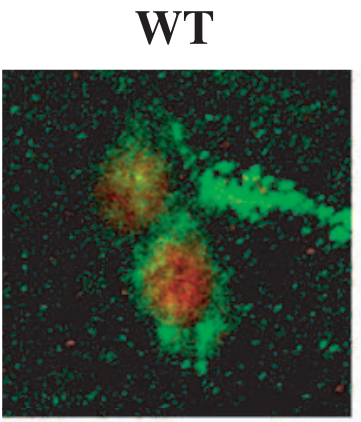

timo1

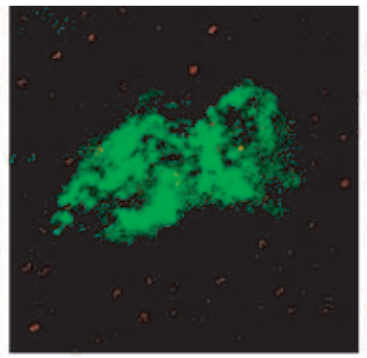

tim01, tim>sgg

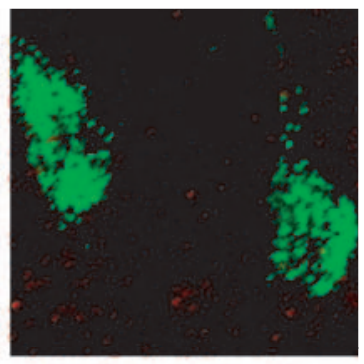

B

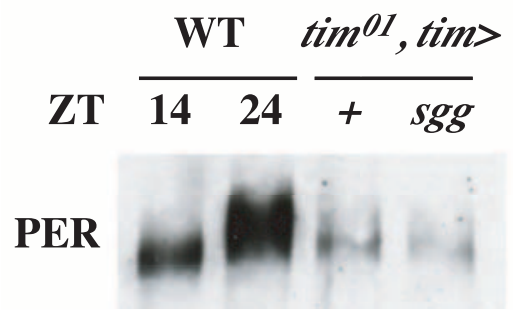

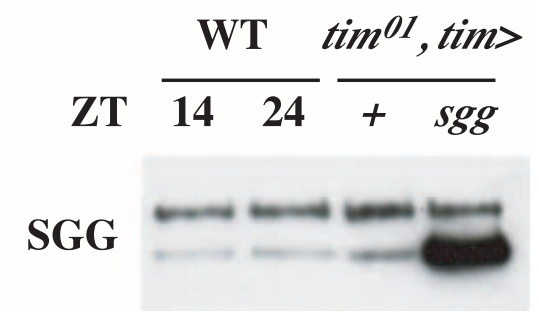

Figure 7. Overexpression of $s g g$ has no effect on PER localization in the absence of tim. $A$, LNs of third instar larval brains were stained for PER (red) and PDF (green), as described in Figure 1. Genotypes analyzed were wild type (WT) at ZT23 (left), tim ${ }^{01}$ (middle), and tim ${ }^{01}$, tim(UAS) - gal4/tim ${ }^{01} ;+/$ uas-sggB (tim ${ }^{01}$, tim > sgg; right). Nuclear PER was only detected in wild-type LNs. The results are representative of $>20$ brain hemispheres per genotype analyzed in at least two independent experiments. $\boldsymbol{B}$, Western blots of extracts of wild-type (ZT14 and ZT2), $\operatorname{tim}^{01}$, tim(UAS) - gal4; +/+ (tim $\left.{ }^{01}, \operatorname{tim}>+\right)$, and tim ${ }^{01}$, tim(UAS) - gal4/ $\operatorname{tim}^{01}$; uas-sggB/+ (tim ${ }^{01}$, tim $>$ sgg) adult heads stained with antibodies to PER (left) and SGG (right). PER levels and mobility in the gel are similar in extracts of $\operatorname{tim}^{01}$ flies regardless of whether sgg is overexpressed. The different bands in the SGG blot are different SGG isoforms. Levels of the upper band (SGG39) are unchanged, but the lower band (SGG10) is overproduced in flies in which uas-sggB is activated by the tim(UAS)- gal4 driver line.

$\mathbf{A}$

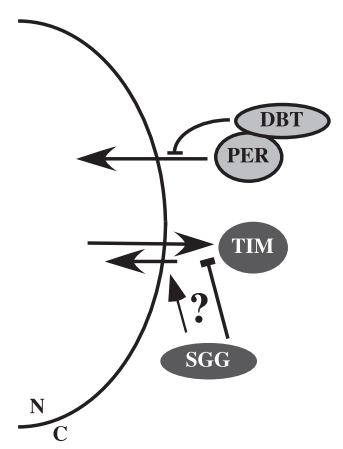

B

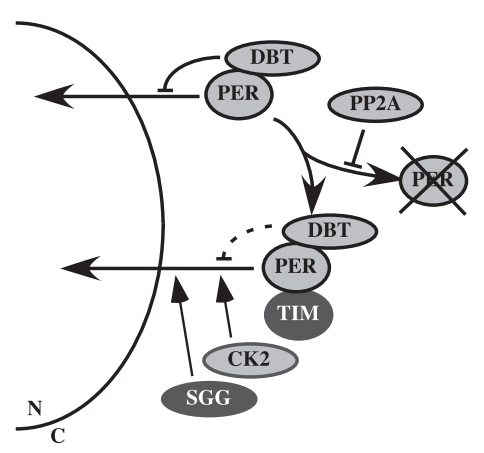

Figure 8. Model describing regulation of PER and TIM nuclear entry. $\boldsymbol{A}$, PER and TIM have opposite intrinsic tendencies. Monomeric PER tends to enter the nucleus (N), but this is inhibited by DBT kinase activity. TIM can enter the nucleus in the absence of PER (Ashmore et al., 2003), but its default state is cytoplasmic (C). SGG phosphorylation of TIM is proposed either to inhibit TIM nuclear export or to promote TIM nuclear entry. $\boldsymbol{B}$, Model for regulation of PER and TIM nuclear entry. PER protein is prevented from entering the nucleus prematurely by DBT, which targets PER for degradation in the absence of TIM. PER accumulates with delayed kinetics in protein phosphatase $2 A(P P 2 A)$ regulatory subunit mutants, indicating that PP2A normally opposes DBT-mediated PER degradation by dephosphorylating PER (Sathyanarayanan et al., 2004). Formation of a PER/TIM complex prevents DBT from phosphorylating PER (Kloss et al., 2001; Ko et al., 2002), thus stabilizing PER. PER and TIM nuclear entry is promoted by SGG, which phosphorylates TIM (Martinek et al., 2001). CK2 activity also promotes PER and TIM nuclear entry, and CK2 can phosphorylate PER in vitro (Lin et al., 2002; Akten et al., 2003). Because PP2A can dephosphorylate PER, it may also play a role in the timing of nuclear entry of PER. DBT and PP2A also determine PER stability in the nucleus, but this is omitted from this model for simplicity.

varying levels of PER overexpression could explain the conflicting experimental results. Our demonstration of a central role for DBT in the nuclear localization of PER in the fly suggests that studies of endogenous PER proteins in naturally occurring clocks might confirm a similar function for CK $1 \epsilon$ in mammals.

PER has been detected in the nucleus without TIM before this study. Shafer et al. (2002, 2004) performed a careful analysis of the timing of PER and TIM nuclear entry in wild-type LNs that indicated that PER was nuclear at times when TIM was only detectable cytoplasmically. Although this suggests that PER can enter the nucleus independently of TIM, the results of Ashmore et al. (2003) permit another interpretation. Although TIM normally accumulates in the cytoplasm of per $^{0}$ mutant pacemaker cells (Hunter-Ensor et al., 1996; Myers et al., 1996), Ashmore et al. (2003) found TIM in the nucleus in per $^{\circ}$ mutants in the presence of nuclear export and proteasome inhibitors. It is therefore likely that TIM normally shuttles in and out of the nucleus and/or is unstable in the nucleus. Even if PER and TIM enter the nucleus as a complex, nuclear PER would accumulate more rapidly than TIM if some nuclear TIM disassociates from PER and is then exported (to recruit more PER) or degraded. Alternatively, given the results of the present study, if DBT activity were to be transiently suppressed by factors other than TIM, PER could enter the nucleus without TIM.

Certainly tim must be included in any clock model given that a null tim mutation stops the molecular clock (Sehgal et al., 1994) and given the dramatic effects of other tim mutations on period length (Rutila et al., 1998b; Rothenfluh et al., 2000a,b). Our model (Fig. $8 \mathrm{~B}$ ) also incorporates data presented in this study, showing that the effects of SGG on the clock require tim. Given the recent report that TIM is required for the mammalian molecular clock (Barnes et al., 2003), it will be interesting to determine its precise function.

\section{Contrasting intrinsic properties of PER and TIM}

Our results reveal that hypophosphorylated PER can enter the nucleus without TIM (Fig. $8 \mathrm{~A}$ ). In contrast, the intrinsic state of TIM appears to be cytoplasmic. Although it is free to enter the nucleus, TIM is rapidly exported and/or degraded in the nucleus, unless it interacts with PER (Fig. 8) (Ashmore et al., 2003). It seems likely that the phosphorylation of TIM by the SGG kinase shifts the balance of TIM localization in favor of the nucleus (Fig. $8 A$ ). Thus, the timing of nuclear localization of PER and TIM probably reflects the divergent effects of DBT and SGG on their substrates. PER has an intrinsic tendency to enter the nucleus but is prevented from doing so via DBT-mediated phosphorylation; TIM tends to stay in the cytoplasm, but SGG makes TIM more likely to enter the nucleus and/or remain there.

In a potentially analogous situation, the transcription of many genes is controlled by both activators and repressors. Such combinations of positive and negative factors have been proposed to 
establish on/off switches in transcription rather than a range of levels of gene expression (Rossi et al., 2000). The opposing tendencies of unmodified PER to enter the nucleus and of unmodified TIM to accumulate in the cytoplasm might similarly help to turn PER and TIM nuclear entry into a switch rather than a gradual event, at least in pacemaker neurons. Indeed, PER and TIM nuclear entry in pacemaker neurons marks a major transition in the behavioral response of flies to light. A light pulse given in the early evening, before PER and TIM enter the nucleus, delays both the molecular clock and the resulting behavior. In contrast, a light pulse at the end of the night, when the proteins are nuclear, phase advances both the clock and behavior (Myers et al., 1996). It is also possible that tight regulation of PER and TIM nuclear entry could help the individual pacemaker neurons within one fly stay synchronized. The results of this study add to our understanding of the intricate regulation of this step in the molecular clock.

\section{Conclusions}

DBT plays two important roles in the Drosophila circadian clock. First, DBT decreases the stability of PER proteins through phosphorylation in the absence of TIM and therefore regulates both the initial cytoplasmic accumulation of PER and the turnover of PER in the nucleus at the end of each cycle as TIM is lost. Second, DBT kinase activity prevents premature accumulation of PER in the nucleus. The function of DBT kinase activity in the cytoplasm establishes the first half of each circadian cycle, separating the phases of transcription and repression of CLK/CYC-activated genes.

TIM also has multiple roles in the Drosophila clock. In wildtype flies, TIM inhibits DBT-dependent phosphorylation of PER (Kloss et al., 2001), and this finding was replicated in S2 cells in culture (Ko et al., 2002). Coproduction of PER and TIM promotes nuclear accumulation of both proteins, supported by SGG-dependent phosphorylation of TIM. Thus, in each of its roles, TIM can be viewed as an important vehicle for controlling PER nuclear localization.

\section{References}

Akashi M, Tsuchiya Y, Yoshino T, Nishida E (2002) Control of intracellular dynamics of mammalian Period proteins by Casein Kinase I epsilon (CKIepsilon) and CKIdelta in cultured cells. Mol Cell Biol 22:1693-1703.

Akten B, Jauch E, Genova GK, Kim EY, Edery I, Raabe T, Jackson FR (2003) A role for CK2 in the Drosophila circadian oscillator. Nat Neurosci 6:251-257.

Allada R, White NE, So WV, Hall JC, Rosbash M (1998) A mutant Drosophila homolog of mammalian clock disrupts circadian rhythms and transcription of period and timeless. Cell 93:791-804.

Ashmore LJ, Sathyanarayanan S, Silvestre DW, Emerson MM, Schotland P, Sehgal A (2003) Novel insights into the regulation of the timeless protein. J Neurosci 23:7810-7819.

Bao S, Rihel J, Bjes E, Fan JY, Price JL (2001) The Drosophila double-times mutation delays the nuclear accumulation of period protein and affects the feedback regulation of period mRNA. J Neurosci 21:7117-7126.

Barnes JW, Tischkau SA, Barnes JA, Mitchell JW, Burgoon PW, Hickok JR, Gillette MU (2003) Requirement of mammalian Timeless for circadian rhythmicity. Science 302:439-442.

Blau J, Young MW (1999) Cycling vrille expression is required for a functional Drosophila clock. Cell 99:661-671.

Bourouis M (2002) Targeted increase in Shaggy activity levels blocks Wingless signaling. Genesis 34:99-102.

Chang DC, Reppert SM (2003) A novel C-terminal domain of Drosophila PERIOD inhibits dCLOCK:CYCLE-mediated transcription. Curr Biol 13:758-762.

Curtin KD, Huang ZJ, Rosbash M (1995) Temporally regulated nuclear entry of the Drosophila period protein contributes to the circadian clock. Neuron 14:365-372.
Cyran SA, Buchsbaum AM, Reddy KL, Lin MC, Glossop NR, Hardin PE, Young MW, Storti RV, Blau J (2003) vrille, Pdp1, and dClock form a second feedback loop in the Drosophila circadian clock. Cell 112:329-341.

Darlington TK, Wager-Smith K, Ceriani MF, Staknis D, Gekakis N, Steeves TDL, Weitz CJ, Takahashi JS, Kay SA (1998) Closing the circadian loop: CLOCK-induced transcription of its own inhibitors per and tim. Science 280:1599-1603.

Edery I, Zwiebel LJ, Dembinska ME, Rosbash M (1994) Temporal phosphorylation of the Drosophila Period protein. Proc Natl Acad Sci USA 91:2260-2264.

Grima B, Lamouroux A, Chelot E, Papin C, Limbourg-Bouchon B, Rouyer F (2002) The F-box protein Slimb controls the levels of clock proteins Period and Timeless. Nature 420:178-182.

Hunter-Ensor M, Ousley A, Sehgal A (1996) Regulation of the Drosophila protein Timeless suggests a mechanism for resetting the circadian clock by light. Cell 84:677-685.

Jia J, Amanai K, Wang G, Tang J, Wang B, Jiang J (2002) Shaggy/GSK3 antagonizes Hedgehog signalling by regulating Cubitus interruptus. Nature 416:548-552.

Kloss B, Price JL, Saez L, Blau J, Rothenfluh A, Wesley CS, Young MW (1998) The Drosophila clock gene double-time encodes a protein closely related to human Casein Kinase I epsilon. Cell 94:97-107.

Kloss B, Rothenfluh A, Young MW, Saez L (2001) Phosphorylation of PERIOD is influenced by cycling physical associations of DOUBLETIME, PERIOD, and TIMELESS in the Drosophila clock. Neuron 30:699-706.

Ko HW, Jiang J, Edery I (2002) Role for Slimb in the degradation of Drosophila Period protein phosphorylated by Doubletime. Nature 420:673-678.

Lin JM, Kilman VL, Keegan K, Paddock B, Emery-Le M, Rosbash M, Allada R (2002) A role for Casein Kinase $2 \alpha$ in the Drosophila circadian clock. Nature 420:816-820

Lowrey PL, Shimomura K, Antoch MP, Yamazaki S, Zemenides PD, Ralph MR, Menaker M, Takahashi JS (2000) Positional syntenic cloning and functional characterization of the mammalian circadian mutation tau. Science 288:483-492.

Martinek S, Inonog S, Manoukian AS, Young MW (2001) A role for the segment polarity gene shaggy/GSK-3 in the Drosophila circadian clock. Cell 105:769-779.

McDonald MJ, Rosbash M (2001) Microarray analysis and organization of circadian gene expression in Drosophila. Cell 107:567-578.

Miyazaki K, Nagase T, Mesaki M, Narukawa J, Ohara O, Ishida N (2004) Phosphorylation of clock protein PER1 regulates its circadian degradation in normal human fibroblasts. Biochem J 380:95-103.

Myers MP, Wager-Smith K, Rothenfluh-Hilfiker A, Young MW (1996) Light-induced degradation of TIMELESS and entrainment of the Drosophila circadian clock. Science 271:1736-1740.

Nawathean P, Rosbash M (2004) The Doubletime and CKII kinases collaborate to potentiate Drosophila PER transcriptional repressor activity. Mol Cell 13:213-223.

Okamura H, Garcia-Rodriguez C, Martinson H, Qin J, Virshup DM, Rao A (2004) A conserved docking motif for CK1 binding controls the nuclear localization of NFAT1. Mol Cell Biol 24:4184-4195.

Panda S, Hogenesch JB, Kay SA (2002) Circadian rhythms from flies to human. Nature 417:329-335.

Preuss F, Fan JY, Kalive M, Bao S, Schuenemann E, Bjes ES, Price JL (2004) Drosophila doubletime mutations which either shorten or lengthen the period of circadian rhythms decrease the protein kinase activity of Casein Kinase I. Mol Cell Biol 24:886-898.

Price JL, Dembinska ME, Young MW, Rosbash M (1995) Suppression of PERIOD protein abundance and circadian cycling by the Drosophila clock mutation timeless. EMBO J 14:4044-4049.

Price JL, Blau J, Rothenfluh A, Abodeely M, Kloss B, Young MW (1998) double-time is a novel Drosophila clock gene that regulates PERIOD protein accumulation. Cell 94:83-95.

Price MA, Kalderon D (2002) Proteolysis of the Hedgehog signaling effector Cubitus interruptus requires phosphorylation by Glycogen Synthase Kinase 3 and Casein Kinase 1. Cell 108:823-835.

Robinow S, White K (1991) Characterization and spatial distribution of the ELAV protein during Drosophila melanogaster development. J Neurobiol 22:443-461.

Rossi FM, Kringstein AM, Spicher A, Guicherit OM, Blau HM (2000) Tran- 
scriptional control: rheostat converted to on/off switch. Mol Cell Biol 6:723-728.

Rothenfluh A, Young MW, Saez L (2000a) A TIMELESS-independent function for PERIOD proteins in the Drosophila clock. Neuron 26:505-514.

Rothenfluh A, Abodeely M, Price JL, Young MW (2000b) Isolation and analysis of six timeless alleles which cause short- or long-period circadian rhythms in Drosophila. Genetics 156:665-675.

Rothenfluh A, Abodeely M, Young MW (2000c) Short-period mutations of per affect a double-time-dependent step in the Drosophila circadian clock. Curr Biol 10:1399-1402.

Rutila JE, Suri V, Le M, So WV, Rosbash M, Hall JC (1998a) CYCLE is a second bHLH-PAS clock protein essential for circadian rhythmicity and transcription of Drosophila period and timeless. Cell 93:805-814.

Rutila JE, Maltseva O, Rosbash M (1998b) The $\operatorname{tim}^{S L}$ mutant affects a restricted portion of the Drosophila melanogaster circadian cycle. J Biol Rhythms 13:380-392.

Sathyanarayanan S, Zheng X, Xiao R, Sehgal A (2004) Posttranslational regulation of Drosophila PERIOD protein by Protein Phosphatase 2A. Cell 116:603-615.

Sehgal A, Price JL, Man B, Young MW (1994) Loss of circadian behavioral rhythms and per RNA oscillations in the Drosophila mutant timeless. Science 263:1603-1606.

Shafer OT, Rosbash M, Truman JW (2002) Sequential nuclear accumula- tion of the clock proteins Period and Timeless in the pacemaker neurons of Drosophila melanogaster. J Neurosci 22:5946-5954.

Shafer OT, Levine JD, Truman JW, Hall JC (2004) Flies by night: effects of changing day length on Drosophila's circadian clock. Curr Biol 14:424-432.

Stanewsky R (2003) Genetic analysis of the circadian system in Drosophila melanogaster and mammals. J Neurobiol 54:111-147.

Suri V, Hall JC, Rosbash M (2000) Two novel doubletime mutants alter circadian properties and eliminate the delay between RNA and protein in Drosophila. J Neurosci 20:7547-7555.

Takano A, Isojima Y, Nagai K (2004) Identification of mPer1 phosphorylation sites responsible for the nuclear entry. J Biol Chem 279:32578-32585.

Toh KL, Jones C, He Y, Eide EJ, Hinz WA, Virshup DM, Ptacek LJ, Fu YH (2001) An hPer2 phosphorylation site mutation in familial advanced sleep phase syndrome. Science 291:1040-1043.

Vielhaber E, Eide E, Rivers A, Gao ZH, Virshup DM (2000) Nuclear entry of the circadian regulator $\mathrm{mPER} 1$ is controlled by mammalian casein kinase I epsilon. Mol Cell Biol 20:4888-4899.

Vosshall LB, Price JL, Sehgal A, Saez L, Young MW (1994) Block in nuclear localization of Period protein by a second clock mutation, timeless. Science 263:1606-1609.

Weber F, Kay SA (2003) A PERIOD inhibitor buffer introduces a delay mechanism for CLK/CYC-activated transcription. FEBS Lett 555:341345 . 